\title{
Macrophage-Mediated Phagocytosis and Dissolution of Amyloid-Like Fibrils in Mice, Monitored by Optical Imaging
}

Tina Richey, ${ }^{*}$ James S. Foster, ${ }^{*}$ Angela D. Williams, ${ }^{*}$ Anna B. Williams, ${ }^{\dagger}$ Alexa Stroh, ${ }^{\ddagger}$ Sallie Macy, ${ }^{*}$ Craig Wooliver, ${ }^{*}$ R. Eric Heidel, ${ }^{\S}$ Siva K. Varanasi, ${ }^{\ddagger}$ Elizabeth N. Ergen, ${ }^{\star}$ Dianne J. Trent, ${ }^{\natural}$ Stephen A. Kania, ${ }^{\natural}$ Stephen J. Kennel, * Emily B. Martin, ${ }^{*}$ and Jonathan S. Wall*

From the Departments of Medicine* and Surgery, ${ }^{\S}$ University of Tennessee Medical Center, Knoxville; the Webb School of Knoxville, ${ }^{\dagger}$ Knoxville; and the Department of Biochemistry, Cellular and Molecular Biology ${ }^{\ddagger}$ and the Department of Biomedical and Diagnostic Sciences, " College of Veterinary Medicine, University of Tennessee, Knoxville, Tennessee

\author{
Accepted for publication \\ January 17, 2019. \\ Address correspondence to \\ Jonathan S. Wall, Ph.D., \\ Amyloidosis and Cancer \\ Theranostics Program, Depart- \\ ment of Medicine, University of \\ Tennessee Medical Center, \\ 1924 Alcoa Hwy, Knoxville, \\ TN 37920. \\ E-mail: jwall@utmck.edu.
}

\begin{abstract}
Light chain-associated amyloidosis is characterized by the extracellular deposition of amyloid fibrils in abdominothoracic organs, skin, soft tissue, and peripheral nerves. Phagocytic cells of the innate immune system appear to be ineffective at clearing the material; however, human light chain amyloid extract, injected subcutaneously into mice, is rapidly cleared in a process that requires neutrophil activity. To better elucidate the phagocytosis of light chain fibrils, a potential method of cell-mediated dissolution, amyloid-like fibrils were labeled with the $\mathrm{pH}$-sensitive dye pHrodo red and a near infrared fluorophore. After injecting this material subcutaneously in mice, optical imaging was used to quantitatively monitor phagocytosis and dissolution of fibrils concurrently. Histologic evaluation of the residual fibril masses revealed the presence of $\mathrm{CD}_{68}{ }^{+}, \mathrm{F} 4 / 80^{+}$, ionized calcium binding adaptor molecule $1^{-}$macrophages containing Congo red-stained fibrils as well as neutrophil-associated proteins with no evidence of intact neutrophils. These data suggest an early infiltration of neutrophils, followed by extensive phagocytosis of the light chain fibrils by macrophages, leading to dissolution of the mass. Optical imaging of this novel murine model, coupled with histologic evaluation, can be used to study the cellular mechanisms underlying dissolution of synthetic amyloid-like fibrils and human amyloid extracts. In addition, it may serve as a test bed to evaluate investigational opsonizing agents that might serve as therapeutic agents for light chain-associated amyloidosis. (Am J Pathol 2019, 189: 989-998; https://doi.org/10.1016/ j.ajpath.2019.01.011)
\end{abstract}

Immunoglobulin light chain-associated amyloidosis (AL) is a plasma cell disorder wherein high concentrations of monoclonal light chain proteins are secreted into the circulation, resulting in the systemic deposition of extracellular amyloid. $^{1-3}$ Amyloid is a complex, compositionally heterogeneous, acellular material composed of light chain fibrils in association with extracellular matrix components and $>20$ serum proteins (notably, serum amyloid $\mathrm{P}$ component and apolipoproteins). ${ }^{4}$ The light chains comprising the fibrils adopt a nonnative structure enabling ordered self-association. ${ }^{5-7}$ The structural modifications of the fibrillar light chains result in the presentation of neoepitopes that can be targeted by monoclonal Igs. ${ }^{8-10}$ Despite the nonnative features of $\mathrm{AL}$ fibrils in pathologic amyloid deposits, phagocytic cells of the innate immune system do not eliminate the material. Consequently, amyloid accumulation is generally progressive, leading to severe organ dysfunction and failure, notably of the heart, nerves, and kidneys. In most clinical cases, patients

\footnotetext{
Supported by The National Institute of Diabetes and Digestive and Kidney Diseases PHS grant DK110038 (J.S.W.); the Department of Medicine, the University of Tennessee Medical Center Cancer Institute (J.S.W.); and the donors contributing to the Cancer Research Gift Fund.

T.R. and J.S.F. contributed equally to this work.

Disclosures: None declared.

Current address of S.K.V., Salk Institute for Biological Studies, La Jolla, CA.
} 
present late with renal or cardiac symptoms. ${ }^{11}$ Treatment for amyloidosis currently focuses on reducing the concentration of the amyloid precursor protein using antiplasma cell chemotherapy or immunotherapy, ${ }^{12-14}$ autologous stem cell transplantation, ${ }^{15,16}$ and protease inhibitors. ${ }^{17}$ In addition, methods to remove tissue amyloid deposits are being developed in hopes that this may promote organ recovery, improve quality of life, and extend patient survival. ${ }^{18-21}$

At present, there are no experimental animal models capable of recapitulating the development, progression, and regression, in response to therapy, of systemic, tissueinfiltrating AL-associated amyloidosis. Injection of patientderived light chain proteins into mice, often in large amounts, has resulted in demonstrable pathology ${ }^{22,23}$; however, these models were technically challenging and relied on nonrenewable, patient-derived resources. The human $\lambda 6$ light chain transgenic murine model, generated by Ward et $\mathrm{al}^{24}{ }^{24}$ resulted in sporadic amyloid formation limited to vacuoles within the stomach wall. As a model tractable for studying amyloidolysis (the dissolution of amyloid) and the efficacy of amyloid-targeting biological agents, human AL-associated amyloid extracts (generally 20 to $100 \mathrm{mg}$ per mouse), isolated from the liver or spleen, have been injected subcutaneously in mice to generate an amyloidoma. ${ }^{25}$ When administered to immunocompetent $\mathrm{BALB} / \mathrm{c}$ mice, these materials were rapidly cleared within approximately 14 days, indicating that, despite being considered inert, components of the mammalian immune system were capable of amyloid removal. ${ }^{25}$ Rapid amyloidosis in this mouse model was associated with a robust humoral and innate immune response associated with neutrophil infiltration and the generation of antibodies to components of the human amyloid material. Subsequent studies with amyloid extract injection into immunocompromised severe combined immunodeficient mice avoided classic T- or B-cell responses, but components of the innate immune response were nonetheless capable of removing the amyloid material, albeit over a longer time frame. ${ }^{25}$

The murine amyloidoma model has been used to visualize, by using small animal positron emission tomography/ computed tomography and single-photon emission computed tomography/computed tomography imaging, the target engagement of radiolabeled monoclonal antibodies (mAbs). ${ }^{10,26,27}$ Treatment of the mice with these mAbs expedited the dissolution of the amyloidoma in severe combined immunodeficient mice, evidenced by a decrease in the wet weight of the residual amyloidoma, excised at necropsy, after 14 or 21 days of treatment. ${ }^{10,25}$ Despite the utility of this mouse model for studying AL amyloid-targeting agents and evaluating the regression of the lesion in response to immunotherapy, it is not amenable to noninvasive quantitative, longitudinal monitoring of amyloid load. Moreover, the model is limited by the availability of large amounts of nonrenewable human amyloid extracts. As an alternative to in vivo studies, phagocytic cells grown in culture have been used to demonstrate uptake of human Alzheimer disease-associated $\beta$ amyloid and AL-associated amyloid in the presence of opsonizing antibodies. ${ }^{21,28,29}$

Herein, we have addressed these limitations by generating a mouse model that uses fluorophore-labeled, renewable, synthetic AL $\lambda 6$ amyloid-like fibrils (approximately $2 \mathrm{mg}$ per mouse) and is amenable to longitudinal, quantitative monitoring of the lesion by optical imaging. In addition, by using dual-fluorophore imaging of individual mice, the dissolution of the fibrilloma was studied concurrently with phagocytosis of the fibrils by cells of the innate immune system using Dylight $800^{21}$ and pHrodo red ${ }^{30}$ fluorophores. This model can be used to study phagocytosis and cellmediated dissolution of amyloid material alone or in the context of investigational opsonizing agents that might serve as novel therapeutics for AL amyloidosis.

\section{Materials and Methods}

\section{rV $\lambda 6$ Wil Amyloid-Like Fibrils}

The recombinant $\lambda 6$ variable domain ( $\mathrm{rV} \lambda 6 \mathrm{Wil}$ ) was synthesized in Escherichia coli and purified, as previously described. ${ }^{31}$ Amyloid-like fibrils were prepared in phosphatebuffered saline with $0.05 \%$ sodium azide, as previously described. ${ }^{32}$ The buffer was exchanged by centrifugation $(2000 \times g, 15$ minutes), followed by the addition of sterile, endotoxin-free phosphate-buffered saline. The presence of fibrils was confirmed using a thioflavin $\mathrm{T}$ assay. ${ }^{32}$ The fibrils were stored at $4^{\circ} \mathrm{C}$ until used.

\section{Preparation of Fluorophore-Labeled rV $\lambda 6$ Wil Amyloid-Like Fibrils}

Aliquots of $\mathrm{rV} \lambda 6 \mathrm{Wil}$ fibrils were labeled, according to manufacturer's instructions, with either the pH-sensitive dye, pHrodo red-STP ester (Life Technologies, Waltham, MA), or the NHS-DyLight800 NHS-ester near-infrared dye (Thermo-Pierce, Waltham, MA). Labeling was performed in $100 \mathrm{mmol} / \mathrm{L}$ sodium bicarbonate buffer, $\mathrm{pH}$ 8.3. For both fluorophores, the unbound dye was removed by buffer exchange after centrifugation at $2000 \times g$ for 15 minutes using sterile phosphate-buffered saline, $\mathrm{pH}$ 7.2.

\section{Electron Microscopy}

Amyloid-like rV $\lambda 6$ Wil fibrils suspended in tris-buffered saline were dried on Formvar-coated copper grids and negatively stained using $4 \%$ uranyl acetate. Images were acquired using a Libra $200 \mathrm{MC}$ transmission electron microscope (Zeiss, Thornwood, NY) with a current of $230 \mu \mathrm{A}$, a voltage of $200 \mathrm{kV}$, and a 1-second exposure.

\section{Flow Cytometry}

Raw 264.7 cells $\left(1 \times 10^{6}\right)$ were incubated with $20 \mu \mathrm{g}$ of $\mathrm{rV} \lambda 6 \mathrm{Wil}$ fibrils (20\% w/w pHrodo red-labeled fibrils) for 2 
hours at $37^{\circ} \mathrm{C}$ and analyzed using an Attune NxT acoustic focusing cytometer (Applied Biosystems, Carlsbad, CA) by gating first for intact cells using forward and side scatter parameters. Fluorescence data were obtained using an excitation at $488 \mathrm{~nm}$, and the emission detected with a 574/26 cutoff filter. The cell population falling within the pHrodo fluorescence-positive gate was determined and is reported as a percentage of the intact cell population.

\section{Optical Imaging of Fluorophore-Labeled rV $\lambda 6$ Wil Fibrils}

A suspension of $\mathrm{rV} \lambda 6 \mathrm{Wil}$ fibrils ( $2 \mathrm{mg} \mathrm{rV} \lambda 6 \mathrm{Wil}$ in $0.2 \mathrm{~mL}$ ), containing $20 \%(\mathrm{w} / \mathrm{w})$ pHrodo red-labeled fibrils, was injected subcutaneously, on the dorsal flank, of female NU/NU mice $(n=4)$. Alternatively, $2 \mathrm{mg}$ of $\mathrm{rV} \lambda 6$ Wil fibrils [with $15 \%$ (w/w) pHrodo red- and 15\% (w/w) DL800-labeled fibrils] was injected into female NU/NU mice $(n=3)$. Mice were imaged under isoflurane anesthesia, in an iBox Scientia small animal optical imaging system (Analytik Jena, Upland, CA), using a Cy5 excitation and emission filter set for pHrodo red and an 800-nm bandpass filter set for DL800 (2-second exposures with $1 \times 1$ binning) over 18 days after injection. Animals were euthanized by isoflurane overdose at day 18 after injection, and the residual fibril masses were harvested and fixed in $10 \%$ buffered formalin ( 24 hours) for histologic examination.

\section{Image Analysis}

The mean raw density of the fibril-associated fluorescence in the pHrodo red and DL800 images was performed initially using a standard elliptical region of interest (ROI) encompassing the fibril mass and a second ROI covering a fibril-free region of the mouse, which served as the mouse-specific background value. The final fluorescence signal intensity was calculated by subtraction of the background mean raw density. The data were also analyzed using a freeform ROI drawn around the borders of the rV $\lambda 6 \mathrm{Wil}$ fibril lesion. The data from the two mouse studies were analyzed by two or three independent reviewers (J.S.W., E.B.M., and A.B.W.) to assess interrater reliability and bias associated with placement of the fibril and control ROIs. The freeform ROI data are presented herein.

\section{Histologic and Immunohistochemical Tissue Staining}

Sections ( $6 \mu \mathrm{m}$ thick) of residual $\mathrm{rV} \lambda 6$ Wil fibril masses, harvested from mice at 18 days after injection, were stained with hematoxylin and eosin (Fisher Scientific, Waltham, MA) or alkaline Congo red solution $(0.8 \% \mathrm{w} / \mathrm{v}$ Congo red, $0.2 \% \mathrm{w} / \mathrm{v}$ $\mathrm{KOH}$, and $80 \%$ ethanol) for 1 hour at room temperature, followed by counterstain with Mayer's hematoxylin for $2 \mathrm{mi}-$ nutes. Consecutive tissue sections were immunostained for the following: human free $\lambda$ light chain (catalog number A10101; Dako, Carpinteria, CA; 1:20,000); CD68 (catalog number ab12512; Abcam, Cambridge, MA; 1:10,000); ionized calcium binding adaptor molecule 1 (Iba-1; catalog number 019-19741; Wako Pure Chemical Industries, Richmond, VA; 1:9000); F4/80 (catalog number MCA497R; Santa Cruz Biotechnology, Dallas, TX; 1:1000); lysosomal associated membrane protein 1 (LAMP1; catalog number sc-19992; Santa Cruz Biotechnology; 1:4000); Ly6G (catalog number sc53515; RB8-8C5; Santa Cruz Biotechnology; 1:5000); myeloperoxidase (catalog number PU496-UP; BioGenex, Fremont, CA; 1:25); and neutrophil elastase (catalog number ab68672; Abcam; 1:3000). Before staining, all tissue sections were subjected to antigen retrieval by boiling in citrate buffer (Dako) for 30 minutes. Slides were developed by addition of peroxidase-conjugated anti-rabbit IgG or anti-rat IgG (ImmPRESS; Vector, Burlingame, CA), as appropriate, for $50 \mathrm{mi}-$ nutes at room temperature, and diaminobenzidine solution (ImmPACT DAB; Vector) for 3 minutes at room temperature.

Photomicrographs of Congo red birefringence were acquired using a Leica DM500 light microscope (Leica Microsystems, Wetzlar, Germany) with a cooled charge-coupled device camera (SPOT RT-Slider; Diagnostic Instruments, Sterling Heights, MI), fitted with cross-polarizing filters. All other images were acquired with a BZ-X700E microscope (Keyence, Atlanta, GA) using bright-field optics or a Texas red filter (for Congo red fluorescence). Typically, a $40 \times$ objective with a $2 \times$ digital zoom and an exposure time of $1 / 40$ seconds was used for bright-field images, unless otherwise noted.

\section{Statistical Analysis}

For quantification of optical imaging data, interrater reliability was assessed by determining the intraclass correlation coefficient, using the two-way random model. Comparison of mean fluorescence intensity data between initial, peak, and final time points was performed by using a paired, two-tailed $t$-test. All statistical analyses were performed using Prism software version 6.07 (GraphPad Software Inc., San Diego, CA) or SPSS software version 25 (IBM Corp., Armonk, NY). Statistical significance was assumed at an $\alpha$ value of 0.05 .

\section{Results}

rV $\lambda 6$ Wil Amyloid-Like Fibrils Are Readily Taken up by RAW 264.7 Cells

Synthetic amyloid-like fibrils were generated using the recombinant $\lambda 6$ variable domain from patient Wil by vigorously shaking the protein at $37^{\circ} \mathrm{C}$. This resulted in a heterogeneous population of fibril structures (Figure 1A). Electron microscopic analysis revealed monodisperse fibril aggregates approximately $15 \mathrm{~nm}$ in diameter and approximately $200 \mathrm{~nm}$ in length observed together with macroscopic aggregates of fibrils that measured $1.2 \mu \mathrm{m}$ along certain axes (Figure 1A).

In these studies, synthetic rV $\lambda 6$ Wil amyloid-like fibrils were mixed with fibrils labeled with an appropriate 

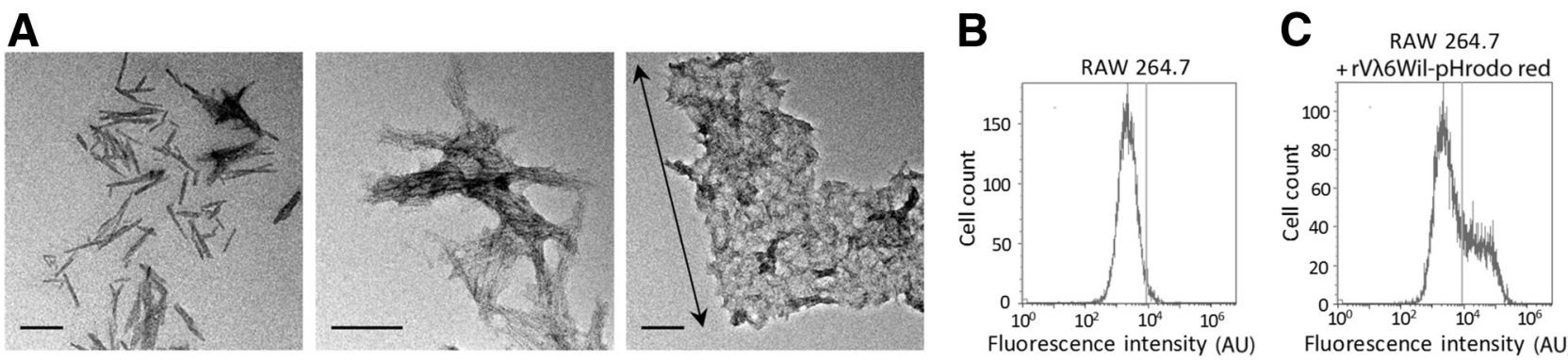

Figure 1 Amyloid-like fibrils composed of $\mathrm{rV} \lambda 6$ Wil are phagocytosed by murine macrophages in culture. A: The structure and varied aggregation states of rV $\lambda 6$ Wil amyloid-like fibrils were determined by electron microscopy. B and C: Incubation of murine RAW 264.7 cells (B) with rV 26 Wil fibrils (20 $\mu \mathrm{g} ; 20 \% \mathrm{w} / \mathrm{w}$ pHrodo red-labeled, $\mathbf{C}$ ) in culture results in $27 \%$ of the cell population staining positive for uptake of fibrils into the acidified phagolysosome. Scale bars: 200 $\mathrm{nm}(\mathbf{A}) ; 1.2 \mu \mathrm{m}(\mathbf{A}$, arrow). AU, arbitrary unit.

fluorophore. The in vitro uptake of $20 \mu \mathrm{g}$ of rV $\lambda 6 \mathrm{Wil}$ fibrils, with $10 \% \mathrm{w} / \mathrm{w}$ pHrodo red-labeled rV $\lambda 6$ Wil fibrils, by RAW 264.7 murine macrophages was monitored by flow cytometry (Figure 1, B and C). The pHrodo red fluorophore fluorescence emission (maximum, $585 \mathrm{~nm}$ ) is enhanced in the acidic microenvironment of the phagolysosome. Cultured RAW 264.7 cells were used to gate side scatter and forward scatter parameters to include only intact cells; in the absence of pHrodo red $\mathrm{rV} \lambda 6 \mathrm{~W}$ il fibrils, they exhibited no fluorescence greater than untreated cells in the red channel (Figure 1B). After a 2-hour incubation with fibrils, $27 \%$ of the cell population exhibited higher than background fluorescence in the Texas red filter channel (emission maximum, approximately $615 \mathrm{~nm}$ ), indicating uptake and acidification of the pHrodo red-labeled fibrils (Figure 1C). These data demonstrate the $\mathrm{pH}$-induced enhancement of pHrodo red fluorescence of the fibrils once engulfed by phagocytic cells.

\section{Phagocytosis of rV $\lambda$ 6Wil Amyloid-Like Fibrils in Vivo}

To further study the phagocytosis of rV $\lambda 6 \mathrm{Wil}$ fibrils in vivo, the uptake, into acidified phagolysosomes, of pHrodo red-labeled $\mathrm{rV} \lambda 6 \mathrm{~W}$ il fibrils was monitored by using optical imaging of hairless, immunocompromised nude (NU/NU) mice (Figure 2). Mice $(n=4)$ were administered, subcutaneously, a 2-mg mass of rV $\lambda 6$ Wil fibrils $(20 \% \mathrm{w} / \mathrm{w}$ pHrodo red fibrils) on the left dorsal flank. On day 1 after injection, no fluorescence was visible on the mouse flank (Figure 2A). In a representative mouse, by day 3 after injection, focal areas of enhanced fluorescence emission were readily visible using optical imaging, within a well-defined fibrilloma (Figure 2A). Serial imaging of the subject demonstrated an increase in the fluorescence emission, reaching a maximum at approximately day 7 after injection, followed by a decrease over the following 11 days, at which point a single focal area of fluorescence intensity remained visible (Figure 2A). At day 18 after injection, the residual fibrilloma was excised post-mortem and appeared as a well-defined lesion associated with the skin, which retained its fluorescence (Figure 2B). The fluorescence intensity associated with the amyloid lesion in each mouse was quantified from optical imaging data using a freeform ROI analysis, and the background-corrected mean raw density was calculated (Figure 2C). Analysis of the optical images is potentially open to subjective bias associated with placement of the ROIs. Therefore, the background-corrected mean raw density data calculated from the two mouse studies were generated by two or three independent reviewers (with only one reviewer in each case familiar with the study design; J.S.W., E.B.M., and A.B.W.), and an intraclass correlation coefficient was calculated to assess bias and consistency. Images analyzed by three reviewers yielded a significant intraclass correlation coefficient of $0.82(P<0.001)$, with a $95 \% \mathrm{CI}$ of 0.62 to 0.92 , and when two reviewers were used, the coefficient increased to $0.94(P<0.001)$, with a $95 \%$ $\mathrm{CI}$ of 0.86 to 0.98 . This analysis indicated that the placement of the ROIs over the fibrilloma and background region of the mouse by the primary reviewer (J.S.W.) of the data was unbiased and consistent with reviewers (E.B.M. and A.B.W.) blinded to the study design.

A significant increase in the fluorescence emission occurred between days 1 and 4 after injection, peaking at approximately day 7 after injection, which represented a rapid onset of phagocytosis of the rV $\lambda 6$ Wil fibrils. The fluorescence emission then decreased between days 7 and 18 after injection (Figure 2C), suggesting that after uptake, dissolution of the fibrils occurred and the fluorophore-labeled breakdown products were no longer present in the acidified compartment. Variability in the mean fluorescence intensity for the group was due to intersubject variation in the time to reach peak fluorescence intensity and the maximum fluorescence emission at the peak, as evidenced when the data for each mouse were plotted (Figure 2D). Despite individual variations, all of the mice exhibited a biphasic change in fluorescence emission (Figure 2D).

\section{Amyloid-Like Fibrils Are Taken up by Macrophages and Multinucleated Giant Cells}

At 18 days after injection, the residual fibrillomas were excised and evaluated histologically (Figure 3). The amyloid-like fibrils were observed in hematoxylin and eosin-stained tissue sections in the s.c. tissue, below the 


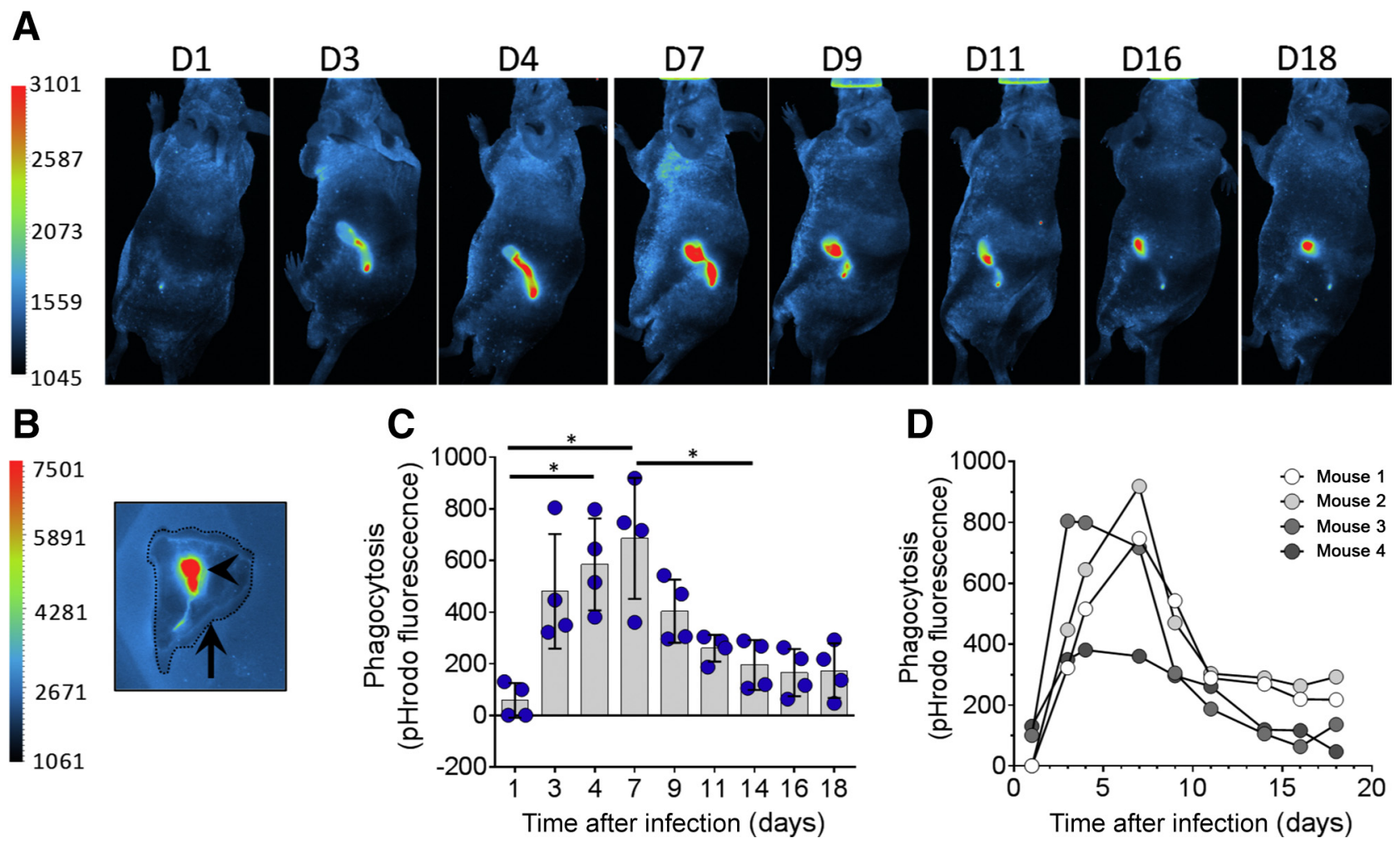

Figure 2 Phagocytosis of rV 6 Wil amyloid-like fibrils in mice monitored by optical imaging. A: Representative (mouse 2) optical images of fluorescence from a mouse with a s.c. $\mathrm{rV} \lambda 6$ Wil fibrilloma containing $20 \% \mathrm{~W} / \mathrm{w}$ pHrodo red-labeled fibrils. Images were captured from day 1 (D1) to day 18 (D18) after injection. B: Optical image of fibrilloma (arrowhead) harvested at 18 days after injection, associated with the skin tissue (arrow). C: Quantification of pHrodo red fluorescence intensity as background-corrected mean raw density. Individual data points are shown at each time point. D: Changes in background-corrected mean raw density pHrodo red fluorescence for each mouse over 18 days after injection. Data are expressed as means \pm SD (C). $* P<0.05$.

cutaneous adipose tissue and panniculus carnosus (muscle) and above the fascial plane (Figure 3A). The pHrodo red-stained $\mathrm{rV} \lambda 6 \mathrm{Wil}$ fibril masses were observed in macrophages (Figure 3B). Multinucleated giant cells, with and without intracellular material reminiscent of unlabeled fibrils, were apparent (Figure 3, C and D). After Congo red staining of the tissues, the amyloid-like fibrils exhibited green-gold birefringence under polarized illumination (Figure 3E), showed characteristic pink congophilia in bright-field images (Figure 3F), and, using the Texas red filter settings, showed Congo red and pHrodo red fluorescence (Figure 3G). Merging of the Congo red bright-field image and red fluorescence indicated that almost all of the amyloid fibril material was cell associated and appeared to be intracellular (Figure 3H).

Immunohistochemical studies confirmed the presence of human $\lambda 6$ light chain-positive fibrils in the macrophages (Figure 3I). The cells containing the amyloid fibrils were immunoreactive with CD68, F4/80, and LAMP1 antibodies, but not Iba-1, indicating that the cells containing the amyloid fibrils were of macrophage lineage (Figure 3, $\mathrm{J}-\mathrm{M})$. Immunostaining for neutrophil-associated markers, elastase, Ly6G, and myeloperoxidase demonstrated an absence of intact neutrophils (Figure 3, N-P); however, amyloid fibril masses and particulates in macrophages were strongly reactive with all three reagents (Figure 3, N and $\mathrm{P})$. These data suggest that although intact neutrophils are not present at day 18 after injection, neutrophil activity in the fibrilloma likely occurred earlier in the lifetime of the mass as fibrils retained remnants of the neutrophil components.

\section{Dual-Fluorophore Imaging Permits Concurrent,} Noninvasive Monitoring of Amyloid Fibril Dissolution and Phagocytosis

To further enhance the murine model, it was considered important to be able to concurrently monitor phagocytosis of the fibrils as well as dissolution of the fibrilloma mass. To this end, $\mathrm{rV} \lambda 6 \mathrm{~W}$ il fibril material was prepared containing $15 \%$ w/w pHrodo red- and 15\% w/w Dylight800-labeled fibrils (Figure 4). The fluorescence emission of both fluorophores was readily detected discriminately by optical imaging in the same fibril-associated lesion. Over the course of 18 days after injection, the fluorescence intensity of the Dylight800-labeled fibrils decreased, indicating a loss of fibrillar material from the mass (Figure 4A). In the same mice, the pHrodo red-associated fluorescence intensity increased to a maximum at day 7 after injection, followed by a decrease to day 18 after injection (Figure 4A), as also 

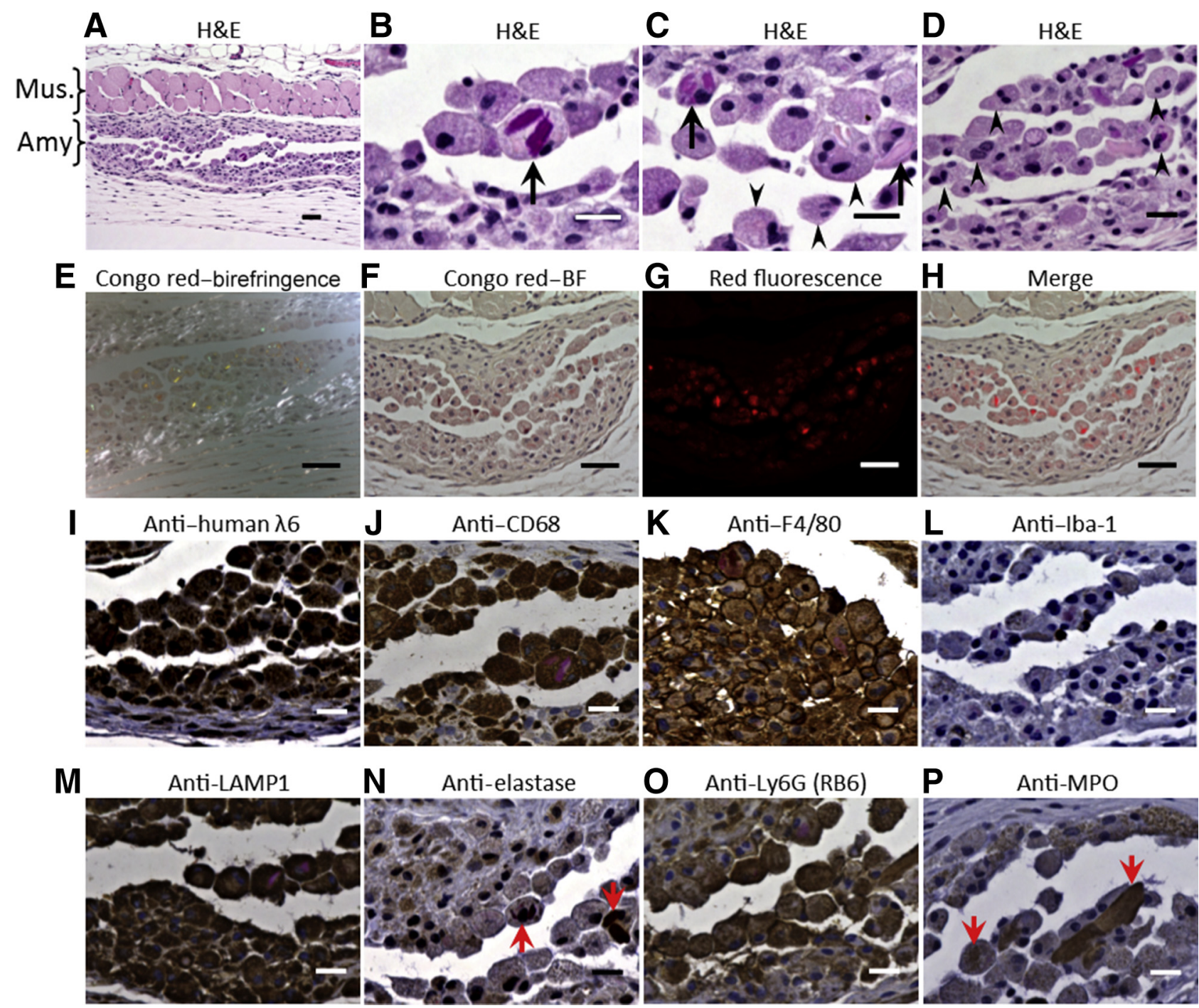

Figure 3 Histologic evaluation of residual fibrilloma shows macrophages with intracellular amyloid. Representative photomicrographs of rV $\lambda 6$ Wil fibrilloma, with $15 \% \mathrm{w} / \mathrm{w}$ pHrodo red-labeled fibrils, harvested 18 days after injection. A: Hematoxylin and eosin (H\&E)-stained tissue section of skin showing panninculus carnosus muscle (Mus.) and amyloid fibril-containing layer (Amy). B-D: H\&E-stained tissue sections showing macrophages with apparent intracellular amyloid (arrows) and multinucleated giant cells (arrowheads). E: Congo red-stained s.c. amyloid viewed under cross-polarized filters showing green-gold birefringence associated with amyloid fibrils. F: Congo red-stained s.c. amyloid viewed under bright-field (BF) illumination showing congophilia associated with amyloid fibrils. G: Congo red-stained s.c. amyloid viewed with epifluorescent illumination (using Texas red emission filter) showing fluorescence of amyloid-like fibrils. H: Merged image of $\mathbf{F}$ and $\mathbf{G}$ indicating that all fluorescent amyloid-like fibrils appear intracellular. I-L: Immunostaining of human $\lambda 6$ light chain, CD68, F4/80, and ionized calcium binding adaptor molecule 1 (Iba-1) in s.c. amyloid. M-P: Immunostaining of lysosomal associated membrane protein 1 (LAMP1), neutrophil elastase, Ly6G (using the RB6 monoclonal antibody clone), and myeloperoxidase (MP0) in s.c. amyloid. Red arrows indicate the presence of intracellular immunostained amyloid-like fibril masses. Scale bars: $50 \mu \mathrm{m}(\mathbf{A}$ and $\mathbf{E}-\mathbf{H}) ; 20 \mu \mathrm{m}(\mathbf{B}-\mathbf{D}$ and I-P).

demonstrated in the previous experiment (Figure 2C). Quantitative analysis of the fibril mass from optical images indicated a significant decrease in the Dylight 800 intensity from day 7 to day 18 after injection (Figure 4, B and C). Over the initial 7 days, the pHrodo red fluorescence of the fibrilloma, indicative of phagocytosis and acidification of the fibrils, increased significantly from baseline and then decreased slightly over the following 11 days (Figure 4D), consistent with the loss of overall fibril mass monitored with Dylight 800 .

\section{Discussion}

The extracellular deposition of AL-associated amyloid results in destruction of organ architecture, leading to compromised organ function notably in the heart, peripheral nerves, and kidneys. Synthetic amyloid-like fibrils may also cause cellular metabolic dysfunction or be cytotoxic, particularly for cardiomyocytes, ${ }^{33-35}$ a pathologic consequence that has also been attributed to the circulating free light chain protein. ${ }^{36-38}$ Cardiac amyloid load, estimated 
A

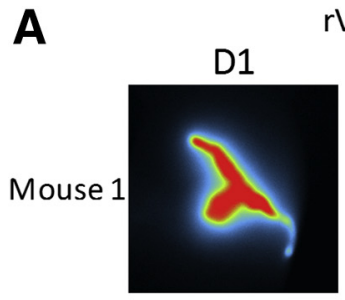

rVג6Wil-Dylight800
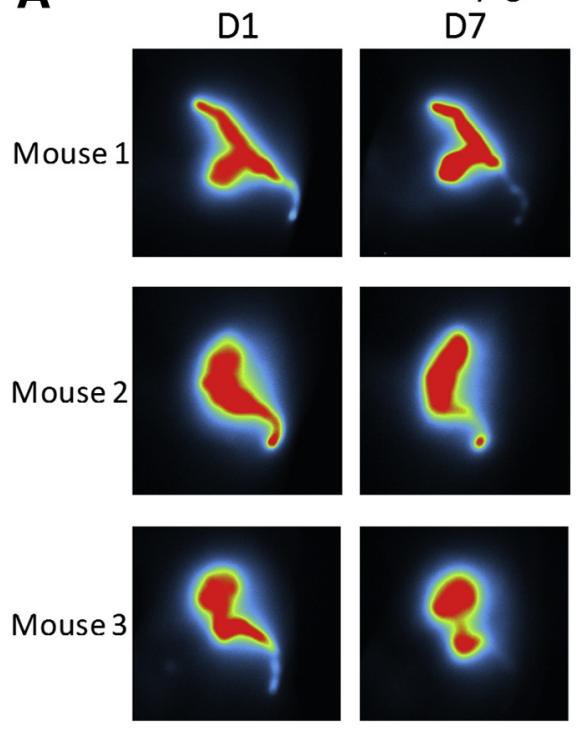

B
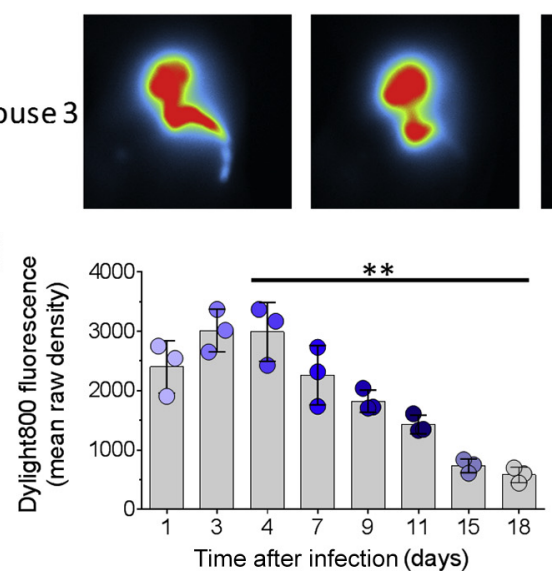

D15
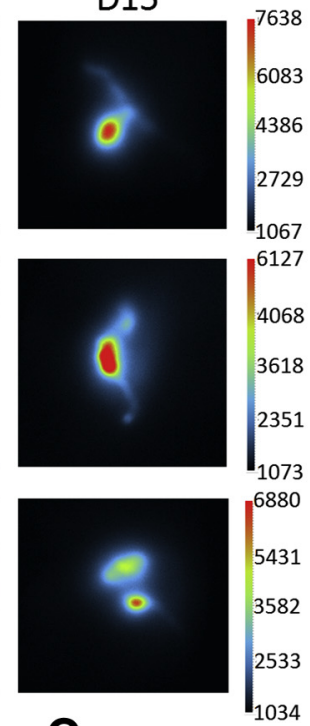

C

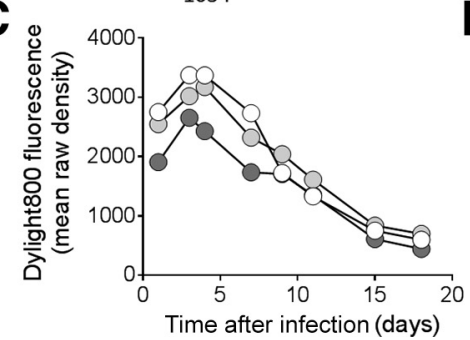

rVA6Wil-pHrodo red
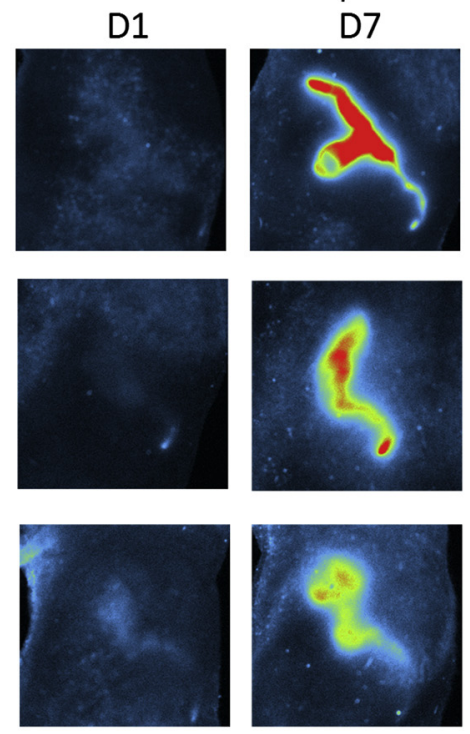

D
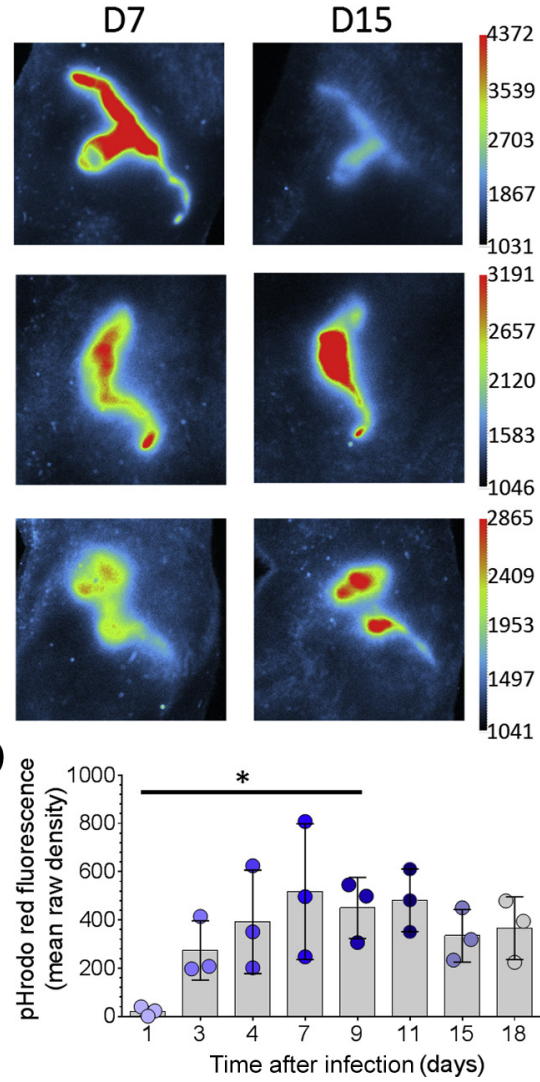

Figure 4 Dual-fluorophore imaging showing phagocytosis and dissolution of rV 6 6Wil fibrilloma in vivo. A: 0ptical imaging of fluorescence from mice with a s.c. rV 6 WWil fibrilloma (15\% w/w Dylight800 and 15\% w/w pHrodo red-labeled fibrils) in each subject on days 1 (D1), 7 (D7), and 15 (D15) after injection. Dylight800 data were scaled to the D1 image, and pHrodo red data were scaled to the D7 images. B-D: Quantification of the Dylight800 fluorescence intensity for the cohort (B), for each mouse in the study (C), and for the pHrodo red fluorescence (D) was determined by region-of-interest analyses and expressed as background-corrected mean raw density. $\mathbf{B}$ and $\mathbf{D}$ : Individual data points are shown at each time point. Data are expressed as means \pm SD (B and $\mathbf{D})$. ${ }^{*} P<0.05,{ }^{* \star} P<0.01$.

histologically from tissue biopsy samples or indirectly using cardiovascular magnetic resonance T1-weighted mapping, has been shown to be an independent predictor of mortality in patients with $\mathrm{AL}$ amyloidosis. ${ }^{39,40}$ Moreover, patients with a lower amyloid load in the heart $(<20 \%$ amyloidoccupied area), who exhibited a positive hematological response to chemotherapy, have a significantly greater overall survival compared with nonresponding patients with a low cardiac AL burden. ${ }^{40}$ Similarly, renal amyloid burden correlates significantly with elevated serum creatinine and reduced estimated glomerular filtration rate, ${ }^{41}$ indicating that greater renal and cardiac amyloid burdens negatively impact organ function and the overall survival in patients with AL.

In light of these observations, there is great interest in effecting removal of tissue amyloid deposits. Complete hematological remission in patients with AL, after chemotherapy or autologous stem cell transplantation, has been shown to reduce amyloid load in the s.c. abdominal fat (evidenced as a decrease in Congo red birefringent material ${ }^{42}$ ); the gastric mucosa $^{43}$; and the liver, ${ }^{44-46}$ with organ-improvement response rates in approximately $20 \%$ of patients, most notably in patients with renal involvement. ${ }^{47}$ In certain patients, this can lead to reversal of amyloid-associated pathologic features and improvement in organ function, such as normalization of cardiac longitudinal strain observed by transthoracic echocardiography. ${ }^{48}$

These clinical observations indicate that reduced amyloid load is associated with improved organ function; however, the mechanism underlying regression of AL-associated amyloid deposits remains unclear. In the absence of data demonstrating significant involvement of phagocytic cells in patient biopsies, the equilibrium hypothesis suggests that, when the free light chain ratio has been normalized, amyloidassociated light chains partition from the fibrils back into the circulation, resulting in passive dissolution of the amyloid fibril. ${ }^{49}$ In contrast, preclinical studies of the clearance of localized or systemic amyloidosis in murine models have indicated the importance of phagocytic macrophages, multinucleated giant cells, and neutrophils. Immunocompetent mice administered human AL amyloid extracts, as s.c. amyloidomas, resolve the material in a process dependent on neutrophil action. Depletion of neutrophils using antigranulocyte receptor $1 \mathrm{mAbs}^{50}$ (although it is now recognized that this also depleted inflammatory monocytes ${ }^{51}$ ) and the use of CD18-knockout mice (with reduced neutrophil activity) delayed the amyloid dissolution. ${ }^{25}$ When the AL 
amyloidomas were induced in severe combined immunodeficient mice, resolution of the material was delayed threefold relative to that in immunocompetent mice; however, amyloidoma resolution in severe combined immunodeficient mice could be expedited by the addition of amyloid-reactive mAbs, which served as opsonins. ${ }^{10,25,26,52}$ In these mice, histologic studies revealed that, in addition to neutrophil activity, Iba- $1^{+}$macrophage infiltration (evidenced at 28 days after injection of the amyloidoma) accompanied the $\mathrm{mAb}$ mediated amyloid clearance. ${ }^{10}$ As an alternative approach, a murine model of systemic serum amyloid protein A-associated amyloidosis was used to demonstrate resolution of hepatosplenic amyloid, in an Fc receptor-dependent manner by macrophages, after administration of an amyloid-reactive Ig. ${ }^{53}$ In addition, passive immunotherapy using amyloid-reactive $\mathrm{mAb}$ induces rapid dissolution of systemic amyloid protein $\mathrm{A}$-associated disease via complement C3-mediated opsonization and the action of multinucleated giant cells,${ }^{54}$ which can avidly take up large amyloid fragments. ${ }^{55}$

Using the model presented herein, we have demonstrated that synthetic amyloid-like fibrils are rapidly taken up into acidified phagolysosomes most commonly associated with macrophages but also present in neutrophils. ${ }^{56}$ The wide range in size of $\mathrm{rV} \lambda 6 \mathrm{Wil}$ particles, ranging from approximately 200-nm fibril shards to macromolecular masses with micrometer dimension, suggests that both neutrophils and macrophages may be involved in the uptake of the synthetic fibrils. The histologic data (Figure 3) suggest the presence of neutrophils in the fibrilloma before day 18 after injection based on the presence of neutrophil elastase and Ly6G reactivity associated with fibril masses and granules in the macrophages (Figure 3, N and $\mathrm{O}$ ), although neutrophils were not directly observed at the time points of the experiments examined herein. Phagocytosis of bacteria and other particulates, including amyloid fibrils, has been evaluated in vitro using the $\mathrm{pH}$-sensitive pHrodo dyes, in which enhanced fluorescence emission is associated with entry of the labeled particle into the phagolysosome. ${ }^{28,30}$ Herein, we have demonstrated, using pHrodo red, that $\mathrm{rV} \lambda 6 \mathrm{Wil}$ fibrils undergo phagocytosis at approximately 3 days after injection and peak at 7 to 11 days after injection. In concert, the fibrilloma is seen to regress based on the decrease in Dylight800 fluorescence emission, which begins approximately 4 days after injection and progresses rapidly (Figure 4B). Using fluorophores with discrete excitation and emission properties has permitted multiplexing and quantitative monitoring of phagocytosis and amyloid dissolution within individual animals.

It was shown, in a quantitative manner, that the innate immune system is capable of rapidly clearing synthetic $\mathrm{AL}$-related amyloid fibrils as well as human AL $\lambda$ amyloid extracts, ${ }^{21}$ although the rate of dissolution is slower for extracts. The importance of macrophages in the clearance of rV $\lambda 6$ Wil fibrils has been established histologically, and the involvement of neutrophils has been implied from the current data. Dissolution of AL-related, amyloid-like fibrils in this mouse model thus involves neutrophils, macrophages, and multinucleated giant cells. The latter are possibly induced during phagocytosis of larger fibrillar particulates. These data suggest a complex process wherein infiltration of neutrophils occurs early, which may result in phagocytosis of smaller fibril particles. Histologic data further indicate the liberation of elastase and myeloperoxidase by neutrophils - both of which were identified as immunohistochemically associated with fibrillar material (Figure 3, N and P). Both are components of neutrophil extracellular traps (NETs), which suggests that neutrophil death in the fibrilloma may have occurred via NETosis ${ }^{57}$; however, other mechanisms, including necrosis, apoptosis, and autophagy, are also possible. This early infiltration is followed by, or occurs in concert with, phagocytosis of amyloid fibrils by macrophages (Figure 3B) and formation of multinucleated giant cells in response to the presence of large fibril particles (Figure 3, C and D). The macrophages persist in the fibrilloma for $>18$ days after injection, during which time the fibril mass is decreasing (Figure 3).

Studies are underway to examine in detail the kinetics of cellular infiltration into the fibrilloma to better characterize the process and more definitively determine the relative contributions of neutrophils and macrophages to phagocytosis and removal of the fibril mass. The current studies are being extended to include ALK and AL $\lambda$ amyloid extracts, which are compositionally more complex, ${ }^{58}$ containing a myriad of accessory molecules in addition to the light chain fibrils; exhibit structurally larger particles; and are generally more resistant to dissolution. ${ }^{21}$ A greater understanding of cellmediated dissolution of AL-associated amyloid will allow us to more effectively recapitulate the process of amyloid removal, thus facilitating development of novel techniques for removal of tissue amyloid from patients. Dual-fluorophore imaging of localized AL amyloid will allow longitudinal monitoring of this process and quantitative evaluation of novel opsonizing agents ${ }^{19,59}$ and pretargeting immunotherapies. ${ }^{21}$

\section{Acknowledgments}

We thank Jim Wesley for tissue preparation and sectioning.

T.R., J.S.F., A.D.W., A.S., S.M., C.W. and D.J.T. performed the experiments; R.E.H. performed statistical analyses; J.S.F., R.E.H., E.N.E., S.A.K., S.J.K., E.B.M., and J.S.W. wrote, revised, and finalized the manuscript; T.R., J.S.F., S.K.V., and J.S.W. designed the studies; A.B.W., R.E.H., E.B.M., and J.S.W. analyzed data; and T.R. and J.S.F. share co-first authorship for equal contributions to and execution of the studies.

\section{References}

1. Dispenzieri A, Gertz MA, Buadi F: What do I need to know about immunoglobulin light chain (AL) amyloidosis? Blood Rev 2012, 26 : 137-154 
2. Merlini G, Bellotti V: Molecular mechanisms of amyloidosis. N Engl J Med 2003, 349:583-596

3. Wechalekar AD, Gillmore JD, Hawkins PN: Systemic amyloidosis. Lancet 2016, 387:2641-2654

4. Dogan A: Amyloidosis: insights from proteomics. Annu Rev Pathol 2017, 12:277-304

5. Raffen R, Dieckman LJ, Szpunar M, Wunschl C, Pokkuluri PR, Dave P, Wilkins Stevens P, Cai X, Schiffer M, Stevens FJ: Physicochemical consequences of amino acid variations that contribute to fibril formation by immunoglobulin light chains. Protein Sci 1999, 8: 509-517

6. Khurana R, Gillespie JR, Talapatra A, Minert LJ, Ionescu-Zanetti C, Millett I, Fink AL: Partially folded intermediates as critical precursors of light chain amyloid fibrils and amorphous aggregates. Biochemistry 2001, 40:3525-3535

7. Velazquez-Lopez I, Valdes-Garcia G, Romero Romero S, Maya Martinez R, Leal-Cervantes AI, Costas M, Sanchez-Lopez R, Amero C, Pastor N, Fernandez Velasco DA: Localized conformational changes trigger the $\mathrm{pH}$-induced fibrillogenesis of an amyloidogenic lambda light chain protein. Biochim Biophys Acta Gen Subj 2018, 1862:1656-1666

8. O'Nuallain B, Allen A, Kennel SJ, Weiss DT, Solomon A, Wall JS: Localization of a conformational epitope common to non-native and fibrillar immunoglobulin light chains. Biochemistry 2007, 46: $1240-1247$

9. O'Nuallain B, Murphy CL, Wolfenbarger DA, Kennel SJ, Solomon A, Wall JS. The amyloid-reactive monoclonal antibody 111F4 binds a cryptic epitope on fibrils and partially denatured immunoglobulin light chains and inhibits fibrillogenesis. Amyloid and Amyloidosis. Edited by Grateau G, Kyle RA, Skinner M. Tours, France: CRC Press, 2005. pp. 482-484

10. Wall JS, Kennel SJ, Williams A, Richey T, Stuckey A, Huang Y, Macy S, Donnell R, Barbour R, Seubert P, Schenk D: AL amyloid imaging and therapy with a monoclonal antibody to a cryptic epitope on amyloid fibrils. PLoS One 2012, 7:e52686

11. Martinez-Naharro A, Hawkins PN, Fontana M: Cardiac amyloidosis. Clin Med 2018, 18:s30-s35

12. Comenzo RL, Steingart RM, Cohen AD: High-dose melphalan versus melphalan plus dexamethasone for AL amyloidosis. N Engl J Med 2008, 358:92

13. Sanchorawala V, Wright DG, Seldin DC, Falk RH, Finn KT, Dember LM, Berk JL, Quillen K, Anderson JJ, Comenzo RL, Skinner M: High-dose intravenous melphalan and autologous stem cell transplantation as initial therapy or following two cycles of oral chemotherapy for the treatment of $\mathrm{AL}$ amyloidosis: results of a prospective randomized trial. Bone Marrow Transplant 2004, 33:381-388

14. Sidiqi MH, Gertz MA: Daratumumab for the treatment of $\mathrm{AL}$ amyloidosis. Leuk Lymphoma 2018, [Epub ahead of print] doi:10.1080/10428194.2018.1485914s

15. Chaulagain CP, Comenzo RL: How we treat systemic light-chain amyloidosis. Clin Adv Hematol Oncol 2015, 13:315-324

16. D'Souza A, Dispenzieri A, Wirk B, Zhang MJ, Huang J, Gertz MA, Kyle RA, Kumar S, Comenzo RL, Peter Gale R, Lazarus HM, Savani BN, Cornell RF, Weiss BM, Vogl DT, Freytes CO, Scott EC, Landau HJ, Moreb JS, Costa LJ, Ramanathan M, Callander NS, Kamble RT, Olsson RF, Ganguly S, Nishihori T, Kindwall-Keller TL, Wood WA, Mark TM, Hari P: Improved outcomes after autologous hematopoietic cell transplantation for light chain amyloidosis: a Center for International Blood and Marrow Transplant Research study. J Clin Oncol 2015, 33:3741-3749

17. Lamm W, Willenbacher W, Lang A, Zojer N, Muldur E, Ludwig H, Schauer-Stalzer B, Zielinski CC, Drach J: Efficacy of the combination of bortezomib and dexamethasone in systemic AL amyloidosis. Ann Hematol 2011, 90:201-206

18. Edwards CV, Gould J, Langer AL, Mapara M, Radhakrishnan J, Maurer MS, Raza S, Mears JG, Wall J, Solomon A, Lentzsch S: Interim analysis of the phase $1 \mathrm{a} / \mathrm{b}$ study of chimeric fibril-reactive monoclonal antibody $11-1 \mathrm{~F} 4$ in patients with AL amyloidosis. Amyloid 2017, 24:58-59

19. Foster JS, Williams AD, Macy S, Richey T, Stuckey A, Wooliver DC, Koul-Tiwari R, Martin EB, Kennel SJ, Wall JS: A peptide-Fc opsonin with pan-amyloid reactivity. Front Immunol 2017, 8:1082

20. Richards DB, Cookson LM, Barton SV, Liefaard L, Lane T, Hutt DF, Ritter JM, Fontana M, Moon JC, Gillmore JD, Wechalekar A, Hawkins PN, Pepys MB: Repeat doses of antibody to serum amyloid $\mathrm{P}$ component clear amyloid deposits in patients with systemic amyloidosis. Sci Transl Med 2018, 10. pii: eaan3128

21. Wall JS, Williams AD, Foster JS, Richey T, Stuckey A, Macy S, Wooliver C, Campagna SR, Tague ED, Farmer AT, Lands RH, Martin EB, Heidel RE, Kennel SJ: Bifunctional amyloid-reactive peptide promotes binding of antibody $11-1 \mathrm{~F} 4$ to diverse amyloid types and enhances therapeutic efficacy. Proc Natl Acad Sci U S A 2018, 115:E10839-E10848

22. Solomon A, Weiss DT, Pepys MB: Induction in mice of human lightchain-associated amyloidosis. Am J Pathol 1992, 140:629-637

23. Teng J, Turbat-Herrera EA, Herrera GA: An animal model of glomerular light-chain-associated amyloidogenesis depicts the crucial role of lysosomes. Kidney Int 2014, 86:738-746

24. Ward JE, SooHoo P, Toraldo G, Jasuja R, Connors LH, O'Hara C, Seldin DC: Metabolic phenotype in an AL amyloidosis transgenic mouse model. Amyloid 2011, 18 Suppl 1:40-41

25. Hrncic R, Wall J, Wolfenbarger DA, Murphy CL, Schell M, Weiss DT, Solomon A: Antibody-mediated resolution of light chainassociated amyloid deposits. Am J Pathol 2000, 157:1239-1246

26. O’Nuallain B, Hrncic R, Wall JS, Weiss DT, Solomon A: Diagnostic and therapeutic potential of amyloid-reactive $\operatorname{IgG}$ antibodies contained in human sera. J Immunol 2006, 176:7071-7078

27. Wall JS, Kennel SJ, Paulus M, Gregor J, Richey T, Avenell J, Yap J, Townsend D, Weiss DT, Solomon A: Radioimaging of light chain amyloid with a fibril-reactive monoclonal antibody. J Nucl Med 2006, 47: 2016-2024

28. Renz M, Torres R, Dolan PJ, Tam SJ, Tapia JR, Li L, Salmans JR, Barbour RM, Shughrue PJ, Nijjar T, Schenk D, Kinney GG, Zago W: 2A4 binds soluble and insoluble light chain aggregates from AL amyloidosis patients and promotes clearance of amyloid deposits by phagocytosis (dagger). Amyloid 2016, 23:168-177

29. Bard F, Cannon C, Barbour R, Burke RL, Games D, Grajeda H, Guido T, Hu K, Huang J, Johnson-Wood K, Khan K, Kholodenko D, Lee M, Lieberburg I, Motter R, Nguyen M, Soriano F, Vasquez N, Weiss K, Welch B, Seubert P, Schenk D, Yednock T: Peripherally administered antibodies against amyloid beta-peptide enter the central nervous system and reduce pathology in a mouse model of Alzheimer disease. Nat Med 2000, 6:916-919

30. Miksa M, Komura H, Wu R, Shah KG, Wang P: A novel method to determine the engulfment of apoptotic cells by macrophages using pHrodo succinimidyl ester. J Immunol Methods 2009, 342:71-77

31. Wall J, Schell M, Murphy C, Hrncic R, Stevens FJ, Solomon A: Thermodynamic instability of human lambda 6 light chains: correlation with fibrillogenicity. Biochemistry 1999, 38:14101-14108

32. Martin EB, Williams A, Heidel E, Macy S, Kennel SJ, Wall JS: Peptide $\mathrm{p} 5$ binds both heparinase-sensitive glycosaminoglycans and fibrils in patient-derived AL amyloid extracts. Biochem Biophys Res Commun 2013, 436:85-89

33. Levinson RT, Olatoye OO, Randles EG, Howell KG, DiCostanzo AC, Ramirez-Alvarado M: Role of mutations in the cellular internalization of amyloidogenic light chains into cardiomyocytes. Sci Rep 2013, 3:1278

34. Lin Y, Marin-Argany M, Dick CJ, Redhage KR, Blancas-Mejia LM, Bulur P, Butler GW, Deeds MC, Madden BJ, Williams A, Wall JS, Dietz A, Ramirez-Alvarado M: Mesenchymal stromal cells protect human cardiomyocytes from amyloid fibril damage. Cytotherapy 2017, 19:1426-1437

35. McWilliams-Koeppen HP, Foster JS, Hackenbrack N, RamirezAlvarado M, Donohoe D, Williams A, Macy S, Wooliver C, 
Wortham D, Morrell-Falvey J, Foster CM, Kennel SJ, Wall JS: Light chain amyloid fibrils cause metabolic dysfunction in human cardiomyocytes. PLoS One 2015, 10:e137716

36. Guan J, Mishra S, Qiu Y, Shi J, Trudeau K, Las G, Liesa M, Shirihai OS, Connors LH, Seldin DC, Falk RH, MacRae CA, Liao R: Lysosomal dysfunction and impaired autophagy underlie the pathogenesis of amyloidogenic light chain-mediated cardiotoxicity. EMBO Mol Med 2014, 6:1493-1507

37. Guan J, Mishra S, Shi J, Plovie E, Qiu Y, Cao X, Gianni D, Jiang B, Del Monte F, Connors LH, Seldin DC, Lavatelli F, Rognoni P, Palladini G, Merlini G, Falk RH, Semigran MJ, Dec GW Jr, Macrae CA, Liao R: Stanniocalcin1 is a key mediator of amyloidogenic light chain induced cardiotoxicity. Basic Res Cardiol 2013, 108:378

38. Mishra S, Guan J, Plovie E, Seldin DC, Connors LH, Merlini G, Falk RH, MacRae CA, Liao R: Human amyloidogenic light chain proteins result in cardiac dysfunction, cell death, and early mortality in zebrafish. Am J Physiol Heart Circ Physiol 2013, 305:H95-H103

39. Banypersad SM, Fontana M, Maestrini V, Sado DM, Captur G, Petrie A, Piechnik SK, Whelan CJ, Herrey AS, Gillmore JD, Lachmann HJ, Wechalekar AD, Hawkins PN, Moon JC: T1 mapping and survival in systemic light-chain amyloidosis. Eur Heart J 2015, $36: 244-251$

40. Kristen AV, Brokbals E, Aus dem Siepen F, Bauer R, Hein S, Aurich M, Riffel J, Behrens HM, Kruger S, Schirmacher P, Katus HA, Rocken C: Cardiac amyloid load: a prognostic and predictive biomarker in patients with light-chain amyloidosis. J Am Coll Cardiol 2016, 68:13-24

41. Kuroda T, Tanabe N, Kobayashi D, Wada Y, Murakami S, Nakano M, Narita I: Significant association between renal function and amyloid-positive area in renal biopsy specimens in AL amyloidosis. BMC Nephrol 2012, 13:118

42. van G II, van Rijswijk MH, Bijzet J, Vellenga E, Hazenberg BP: Histological regression of amyloid in AL amyloidosis is exclusively seen after normalization of serum free light chain. Haematologica 2009, 94:1094-1100

43. Katoh N, Matsuda M, Tsuchiya-Suzuki A, Ikeda S: Regression of gastroduodenal amyloid deposition in systemic AL amyloidosis after intensive chemotherapies. Br J Haematol 2011, 153:535-538

44. Katoh N, Matsushima A, Kurozumi M, Matsuda M, Ikeda S: Marked and rapid regression of hepatic amyloid deposition in a patient with systemic light chain (AL) amyloidosis after high-dose melphalan therapy with stem cell transplantation. Intern Med 2014, 53:1991-1995

45. Lovat LB, Persey MR, Madhoo S, Pepys MB, Hawkins PN: The liver in systemic amyloidosis: insights from 123I serum amyloid P component scintigraphy in 484 patients. Gut 1998, 42:727-734

46. Hawkins PN, Richardson S, MacSweeney JE, King AD, Vigushin DM, Lavender JP, Pepys MB: Scintigraphic quantification and serial monitoring of human visceral amyloid deposits provide evidence for turnover and regression. Q J Med 1993, 86:365-374

47. Gertz MA, Kyle RA, Greipp PR: Response rates and survival in primary systemic amyloidosis. Blood 1991, 77:257-262

48. Fitzgerald BT, Bashford J, Scalia GM: Regression of the anatomic cardiac features of amyloid light chain cardiac amyloidosis accompanied by normalization of global longitudinal strain. Case 2017, 1:46-48

49. Safadi S, Saad A, Quint PS, Sethi S, Leung N, Kurtin P, Nasr SH: Disappearance of immunoglobulins from persistent renal amyloid deposits following stem cell transplantation for heavy- and light-chain amyloidosis. Nephrol Dial Transplant 2015, 30:1151-1155

50. Thomas J, Gangappa S, Kanangat S, Rouse BT: On the essential involvement of neutrophils in the immunopathologic disease: herpetic stromal keratitis. J Immunol 1997, 158:1383-1391

51. Schumak B, Klocke K, Kuepper JM, Biswas A, Djie-Maletz A, Limmer A, van Rooijen N, Mack M, Hoerauf A, Dunay IR: Specific depletion of Ly6C(hi) inflammatory monocytes prevents immunopathology in experimental cerebral malaria. PLoS One 2015, 10: e0124080

52. Solomon A, Weiss DT, Wall JS: Therapeutic potential of chimeric amyloid-reactive monoclonal antibody 11-1F4. Clin Cancer Res 2003, 9:3831S-3838S

53. Nystrom SN, Westermark GT: AA-amyloid is cleared by endogenous immunological mechanisms. Amyloid 2012, 19:138-145

54. Bodin K, Ellmerich S, Kahan MC, Tennent GA, Loesch A, Gilbertson JA, Hutchinson WL, Mangione PP, Gallimore JR, Millar DJ, Minogue S, Dhillon AP, Taylor GW, Bradwell AR, Petrie A, Gillmore JD, Bellotti V, Botto M, Hawkins PN, Pepys MB: Antibodies to human serum amyloid $\mathrm{P}$ component eliminate visceral amyloid deposits. Nature 2010, 468:93-97

55. Milde R, Ritter J, Tennent GA, Loesch A, Martinez FO, Gordon S, Pepys MB, Verschoor A, Helming L: Multinucleated giant cells are specialized for complement-mediated phagocytosis and large target destruction. Cell Rep 2015, 13:1937-1948

56. Cech P, Lehrer RI: Phagolysosomal pH of human neutrophils. Blood 1984, 63:88-95

57. Kaplan MJ, Radic M: Neutrophil extracellular traps: double-edged swords of innate immunity. J Immunol 2012, 189:2689-2695

58. Piehl DW, Blancas-Mejia LM, Ramirez-Alvarado M, Rienstra CM: Solid-state NMR chemical shift assignments for AL-09 VL immunoglobulin light chain fibrils. Biomol NMR Assign 2017, 11: $45-50$

59. Foster JS, Koul-Tiwari R, Williams A, Martin EB, Richey T, Stuckey A, Macy S, Kennel SJ, Wall JS: Preliminary characterization of a novel peptide-Fc-fusion construct for targeting amyloid deposits. Amyloid 2017, 24:26-27 